\title{
Positron Emission Profiling study of the diffusion of 3-methylpentane in silicalite-1
}

\author{
Alina O. Koriabkina ${ }^{\mathrm{a}, *}$, Danny Schuring ${ }^{\mathrm{a}}$, Arthur M. de Jong ${ }^{\mathrm{b}}$, and Rutger A. van Santen ${ }^{\mathrm{a}}$ \\ ${ }^{a}$ Laboratory of Inorganic Chemistry and Catalysis, Schuit Institute of Catalysis, Eindhoven University of Technology, P.O.Box 513, 5600 MB \\ Eindhoven, The Netherlands \\ ${ }^{\mathrm{b}}$ Accelerator Laboratory, Schuit Institute of Catalysis, Eindhoven University of Technology, P.O. Box 513, 5600 MB Eindhoven, \\ The Netherlands
}

\begin{abstract}
The influence of the loading on the self-diffusion of 3-methylpentane within silicalite-1 has been studied with Positron Emission Profiling technique. A monotonous decrease in the self-diffusivities of alkane was observed with zeolite loading, which could be approximated by a stretched exponential. As a consequence, the apparent activation energy increases with partial pressure.
\end{abstract}

KEY WORDS: alkane; diffusivity; activation energy of diffusion; silicalite; PEP.

\section{Introduction}

For Sir John Thomas' 70th birthday. In appreciation of his great stimulation and support for the computational modeling and in situ study of zeolitic systems. His style cannot be matched!

For several decades, many studies of the diffusive and adsorptive properties of alkanes in MFI-zeolites have been carried out [1]. These materials are of great interest in the petroleum industry as catalysts for hydrocarbon conversion processes. 3-Methylpentane is one of the main products of the hydroisomerization of $n$-hexane, an important reaction to upgrade the gasoline octane number. Diffusion of the reactant/product mixture might play a significant role in the selectivity of the catalytic process. In our previous study of the $n$-hexane/ isohexane mixture diffusion in silicalite [2], it has been shown that the diffusion of both alkanes is controlled by the concentration of the slow component. Therefore, the concentration dependence of the diffusion coefficient, especially for bulky alkanes (as isohexane) is an important issue. Silicalite, which consists of straight and zigzag channels connected via intersections, was used as a model of the active zeolite matrix. This allsilica zeolite does not contain acid sites, which excludes any possible influence on the diffusion process [3].

Nowadays, a number of techniques are available to study the diffusion of hydrocarbons in zeolites [1]. In this study, the Positron Emission Profiling technique (PEP) [4] was applied, which is based on the use of radio-labeled molecules to measure self-diffusion coefficients. Adsorption-kinetic techniques that use radiolabeled components for the purpose of quantitative phase analysis have been frequently described in the past

\footnotetext{
* To whom correspondence should be addressed. E-mail: a.koryabkina@TUE.NL
}

[5]. PEP that is used here is a one-dimensional analog of Positron Emission computed Tomography (PET) that has been applied to the study of catalytic systems [6]. In principle, PEP is a technique capable of measuring the concentration profiles of radio-labeled molecules at different positions inside the reactor.

In the literature, there are five different phenomena mentioned that describe the concentration dependence of self-diffusion in the zeolites [7]: (1) the diffusivity decreases monotonically with occupancy; (2) the diffusivity is constant up to medium pore filling, and at high loadings, a decrease in the diffusivity can be observed; (3) at low concentration, the diffusivity monotonically increases and then has a constant value with further increase in the loading; (4) the diffusivity has a maximum at certain pore filling; and (5) the diffusion coefficient monotonically increases with the concentration. Usually, the concentration dependence of type-I is observed for saturated hydrocarbons in silicalite.

Frequency Response [8] and Square Wave [9] methods have been used to measure the decrease in the diffusivity for $n$-hexane in silicalite with loading, which is in agreement with the results obtained by Micke et al. from the sorption uptake kinetics [10]. The $n$ hexane diffusivity in silicalite-1 reported therein is independent of concentration, but a linear concentration dependence was found for the self-diffusivity. For smaller alkanes like methane, ethane, and propane in silicalite NMR has shown the self-diffusivities to reduce with the concentration [11]. Kinetic Monte Carlo simulations performed by Coppens et al. [12] have also shown that in MFI-type zeolites, the self-diffusivity strongly depends on the loading and decreases several orders of magnitude as the occupancy of silicalite increases. Similar behavior was found for the selfdiffusion of linear and branched alkanes in various zeolites using MD simulations [13]. 
In the past years, several theories have been developed, trying to derive an expression that would be able to predict and describe the concentration dependence of the diffusion in zeolites. In these theories, self-diffusion is assumed to proceed via the sequence of the activated jumps between the adsorption sites. One of the first predictions had been made in 1960 using mean-field theory [14], according to which a linear decrease with the pore occupancy can be observed. The self-diffusivity would be proportional to the fraction of unoccupied adsorption sites $1-\theta$, reflecting the probability that a neighbor adsorption site is free for the molecule to jump. Later, Monte Carlo simulations revealed deviations from this linear dependence [12,15-17], showing a stronger decrease in the self-diffusivity with pore occupancy for MFI-type zeolites. In these studies, the topology of this particular zeolite has been taken into account. Correlation effects were found to be more significant for the molecular diffusion in the poorly connected zeolite matrixes such as silicalite, so that the diffusion coefficient decreased faster with the zeolite loading [12]. A unified model based on the random walk/hopping mechanism has been offered by Chen and Yang [18] capable of describing both decreasing and increasing concentration dependencies.

In the present experimental study of the concentration influence on self-diffusion of 3-methylpentane in silicalite, we have tried to establish this dependence and analyze it with existing models. Factors influencing the apparent activation energy of diffusion have also been studied.

\section{Experimental section}

\subsection{Experimental setup}

The PEP technique is a radiochemical in situ method using $\beta^{+}$-emitting isotopes. In our experiments, ${ }^{11} \mathrm{C}$ labeled hydrocarbons are used. The ${ }^{11} \mathrm{C}$ isotope is produced via irradiation of a nitrogen target with $12 \mathrm{MeV}$ protons from the $30 \mathrm{MeV}$ AVF Cyclotron of the Eindhoven University of Technology.

The radio-labeled hydrocarbons $\left(C_{1}-C_{6}\right.$ alkanes) are produced from ${ }^{11} \mathrm{CO}$ and nonlabeled 1-pentene using a homologation reaction. Detailed information about this process can be found in the article of Cunningham et al. [19]. The hydrocarbon of interest (3-methylpentane) is separated from the reaction products using a chromatographic column.

During the experiments, a constant flow of nonlabeled hydrocarbon (3-methylpentane) in a carrier gas (hydrogen) was fed in a plug flow reactor containing the zeolite sample. The partial pressure of the alkane was set by varying the ratio between the alkane gas flow and hydrogen flow (with the total flow kept constant and equal to $150 \mathrm{NmL} / \mathrm{min}$ ) using a Bronkhorst CEM system. Under zero partial pressure conditions, only the hydrogen as a carrier gas was fed into the reactor with the flow rate of $150 \mathrm{NmL} / \mathrm{min}$. In order to measure the self-diffusivity of the alkane, a pulse containing approximately $10^{-15}$ moles of the ${ }^{11} \mathrm{C}$-labeled hydrocarbon of interest is injected in the flow passing through the reactor. The development of the pulse (change of the radio-labeled concentration profile in time) is monitored using a detection system based on the principal described below.

The radioactive nuclei of ${ }^{11} \mathrm{C}$ decay with a half-life of $20.4 \mathrm{~min}$, emitting a positron. The annihilation of positrons with electrons from the surrounding matter results in the creation of pairs of $\gamma$-photons emitted in opposite directions. The position at which the annihilation takes place (which is the position of the radiolabeled molecule) is determined by coincident detection of the photon pair. In this way, the PEP technique is capable of monitoring the concentration of labeled molecules along the cylindrical axis of the reactor (figure 1) with a time resolution of $1 \mathrm{~s}$ and a spatial resolution of $3 \mathrm{~mm}$. Each plot on figure 1 shows a changing concentration of radio-labeled alkane at a certain detection position in time. The bed length of the reactor was $30 \mathrm{~mm}$, so 10 detection positions were involved. A widening of the concentration profile occurs when the pulse moves along the reactor bed. The asymmetric shape of the profiles indicates that intracrystalline diffusion is dominant. A detailed description of the detection system can be found in the work of Mangnus et al. [20].

The isotherms of adsorption for 3-methylpentane in silicalite have been measured using a Balzers Quadrupole mass spectrometer system QMG-420 at 403-483 K. The silicalite sample was exposed to a hydrocarbon/ hydrogen stream until an equilibrium between the gas and adsorbed phase was reached, which was monitored using the mass spectrometer. The system was considered to be in equilibrium when the signals of the hydrogen and alkane masses were constant. After equilibration, the flow of the hydrocarbon was switched off, resulting in the desorption of the adsorbed species. The total

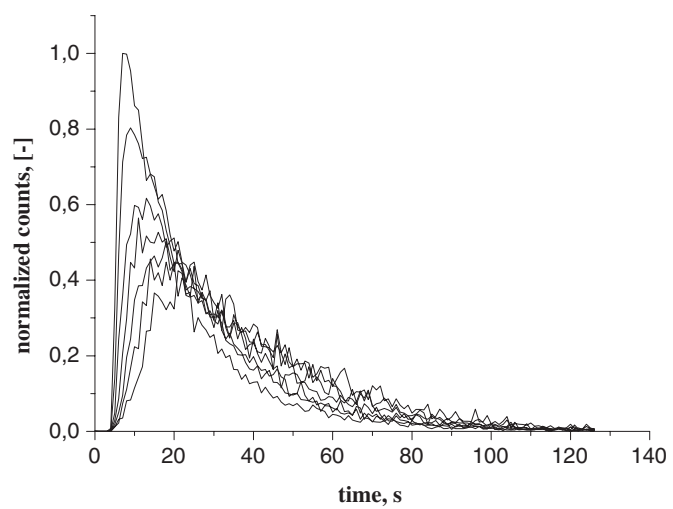

Figure 1. Experimental data: concentration profiles for 7 reactor positions, 3-methylpentane in silicalite, $T=443 \mathrm{~K}$. 
amount of desorbed alkane was then calculated from the integration of a calibrated signal of 3-methylpentane mass over desorption time. The alkane loading at a given partial pressure and temperature is determined as the amount of the desorbed hydrocarbon per gram of the zeolite. The zeolite pore occupancy $\theta$ was calculated as a ratio between the loading under given conditions and the maximum loading.

The 3-methylpentane used in the experiments has been obtained from Aldrich (99.9\% purity) and was used as received.

\subsection{Zeolite samples}

The silicalite-1 sample used has been kindly provided by the Shell Research and Technology Center, Amsterdam. Scanning electron microscopy showed that it consisted of regular coffin-shaped crystals with an average size of $(150 \times 50 \times 30 \mu \mathrm{m})$. The bed porosity was determined to be $\varepsilon=0.44$. Prior to experiments, the zeolite had been activated in a hydrogen stream for $2 \mathrm{~h}$ at $723 \mathrm{~K}$.

\section{Modeling}

In order to interpret the data provided by the PEP experiments, an appropriate mathematical model is used to describe the transport through the zeolite reactor bed. The model used to describe the molecular transport in steady-state experiments (TEX-PEP experiments [2]) was applied to the pulse experiments, but with different initial conditions. A detailed description of the model that is basically a modification of the equations used by Noordhoek et al. [21], and more information about its application can be found in the work of Schuring et al. [2]. The model used to analyze the pulse experiments will be only briefly discussed below.

In the zeolite bed, transport of molecules occurs via convection and axial diffusion in the bed, adsorption/ desorption at the crystal surface, and diffusion inside the pores of the crystals. The zeolite crystals are considered to have a spherical shape. This approximation is commonly made in the literature and has been shown to be quite reasonable [1]. The value of the radius $(R=25 \mu \mathrm{m})$ is chosen so as to ensure that the sphere has the same external surface area to volume ratio as the coffin-shaped zeolite crystals [1].

Mass transfer through the bed, resulting from diffusion, convection, and mass transfer to the zeolite crystals, can be described by

$$
\frac{\partial c_{\text {gas }}}{\partial t}=D_{\text {ax }} \frac{\partial^{2} c_{\text {gas }}}{\partial z^{2}}-v_{\text {int }} \frac{\partial c_{\text {gas }}}{\partial z}+\frac{3(1-\varepsilon)}{R \varepsilon} N_{c}
$$

In this equation, $z$ is the coordinate along the catalyst bed, $c_{\text {gas }}$ is a gas-phase concentration in the catalyst bed,
$D_{\text {ax }}$ is the axial diffusion coefficient, calculated from the molecular diffusion coefficient of the component. $v_{\text {int }}$ is the interstitial velocity, which is defined as

$$
v_{\text {int }}=\frac{v_{\text {sup }}}{\varepsilon}
$$

in which $v_{\text {sup }}$ is the gas flow velocity in the empty reactor. $R, D_{\mathrm{ax}}, v_{\text {int }}$, and $\varepsilon$ are fixed parameters, which can be determined experimentally or estimated.

The term $N_{c}$ is the mass flux through the boundary of the zeolite crystal. It is determined by the rate-limiting step for the adsorption/desorption at the crystal boundary. Diffusion through the laminar fluid film surrounding the particles can be neglected, as this process is much faster than diffusion inside the zeolite crystal. Adsorption/desorption process at the crystal boundary was discussed in our earlier study [2] and it was shown that it is fast compared to the diffusion inside the zeolite crystal, and therefore, the term $N_{c}$ is determined by diffusion to the crystal boundary:

$$
N_{c}=-\left.D_{c} \frac{\partial c_{x}}{\partial x}\right|_{x=R}
$$

where $c_{x}$ is the adsorbed phase concentration inside the particle, and $D_{c}$ is the intracrystalline diffusivity. Thus, the boundary equation at the crystal surface is expressed by a simple equilibrium condition:

$$
c_{x}(R, z, t)=K_{a} \times c_{\text {gas }}(z, t)
$$

where $K_{a}$ is the adsorption equilibrium constant (should not be confused with the Langmuir adsorption constant). In the experiments with an empty zeolite, due to a very low concentration of the radio-labeled molecules injected, the Henry's regime is fulfilled. Then, the equation (4) becomes mathematically the same as Henry's law.

Mass transport in the pores of the zeolite crystal only occurs by diffusion:

$$
\frac{\partial c_{x}}{\partial t}=D_{c}\left[\frac{\partial^{2} c_{x}}{\partial x^{2}}+\frac{2}{x} \frac{\partial c_{x}}{\partial x}\right]
$$

In this equation, $x$ is the radial coordinate in the crystal and $D_{c}$ is the intracrystalline diffusion coefficient.

Before the pulse is injected, the zeolite reactor bed does not contain any radio-labeled molecules, which provides the initial conditions:

$$
c_{\text {gas }}(z, 0)=0, \quad c_{z, 0^{-}}=c_{0} \cdot f(t) .
$$

Here, $c_{z, 0^{-}}$is the concentration of radio-labeled molecules just before the zeolite bed entrance, $c_{0}$ is the initial injected concentration of radio-labeled molecules, and $f(t)$ is a Gaussian pulse shape function. 


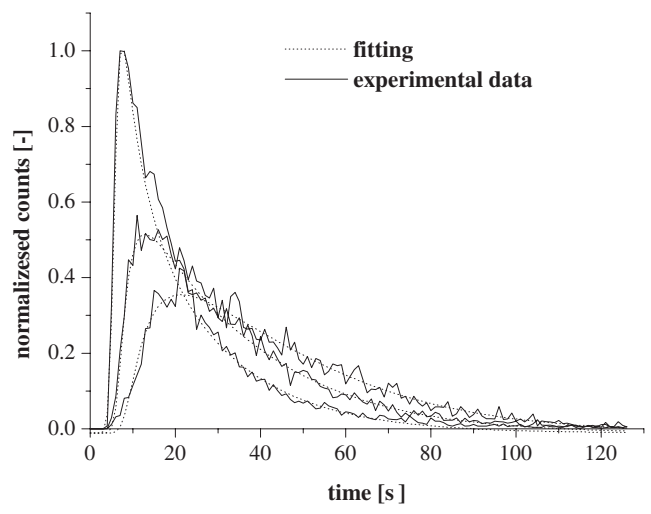

Figure 2. Example fit of concentration profiles at 3 reactor positions for 3-methylpentane in silicalite, $T=443 \mathrm{~K}$.

The equations described above are solved using the numerical method of lines [22]. Fitting the modeled concentration profiles to the measured ones yields a value of the unknown parameters such as the adsorption constant $K_{a}$ and intracrystalline diffusivity $D_{c}$. An example fit of the experimental data is shown in figure 2. In our previous study [2], we have shown that the presence of intracrystalline diffusion limitations has a significant influence on the shape of the concentration profiles causing the asymmetry: the lower the diffusivity $D_{c}$, the stronger the tailing of the concentration profile. Parameter $K_{a}$ is related to the delay of the profiles: the smaller the delay, the lower the value of $K_{a}$. Thus, the parameters $D_{c}$ and $K_{a}$ play different roles in the description of the concentration profile. This provides a possibility to extract reasonable data on self-diffusivities from the fitting of the model to the PEP experiments. Modeling translated the experimental error into the following error in the fitted parameters. The diffusion coefficients $D_{c}$ and $K_{a}$ have been determined from the fit with the error of approximately $10 \%$.

\section{Results and discussion}

\subsection{Influence of the concentration on the diffusivity}

The self-diffusivities of 3-methylpentane have been measured at temperatures from 403 to $483 \mathrm{~K}$ at hydrocarbon partial pressures from 0 to $4.5 \mathrm{kPa}$ with a constant total flow rate of $150 \mathrm{NmL} / \mathrm{min}$. In the experiments at zero loading, the pulses of ${ }^{11} \mathrm{C}$-labeled alkane have been injected in the flow of hydrogen passing through the reactor.

\subsubsection{Isotherms of 3-methylpentane adsorption in silicalite}

The loadings under given conditions (temperature, partial pressure) have been determined from the isotherms of adsorption measured with nonlabeled 3methylpentane using a mass spectrometer (see Section
2.1). In principle, pulse experiments using the PEP technique allow to determine zeolite pore occupancy from the retention time of the alkane in the catalyst bed, calculated using the theory of statistical moments, as was done in the work of van Santen et al. [23]:

$$
\theta(p)=\frac{\tau_{0}-\tau_{p}}{\tau_{0}}
$$

In this equation, $\tau_{0}$ and $\tau_{p}$ are the retention times of the alkane in the empty zeolite with hydrogen as a carrier gas and zeolite saturated at the given hydrocarbon partial pressure respectively. Unfortunately, this approach does not provide very accurate data. The loadings determined from the PEP data using expression 7 and those obtained from the mass spectrometer experiments are shown in figure 3. Using expression 7 to determine the pore occupancy $\theta$ provides a larger error, probably because some approximations used are not completely fulfilled here. Even though there is an agreement between the data from both methods, due to the higher error in the pore occupancy $\theta$ determined by the method of statistical moments from expression 7, it would be inconvenient to use these as parameter to establish the influence of the loading on the diffusion.

The adsorption isotherms have been fitted using a Langmuir curve (figure 3). Although the dual-site Langmuir model is applied to describe the isotherm of adsorption for isohexanes [24], we used a simple Langmuir model because the partial pressure conditions of this study were significantly lower compared to those required to observe an inflection in the isotherm.

Fitting the data provided the equilibrium constants of adsorption and the maximum loading $\left(C^{\max }=\right.$ $0.75 \pm 0.05 \mathrm{mmol} / \mathrm{g}$ ). This value is close to approximately four molecules per unit cell, which is predicted by CBMC simulations [24] as the loading for the inflection to occur. This is in good agreement with Cavalcante et al. [25], who measured $0.7 \mathrm{mmol} / \mathrm{g}$ as maximum loading for 3-methylpentane in silicalite using a gravimetric technique.

From the temperature dependence of the fitted adsorption constant (figure 4), the enthalpy of adsorp-

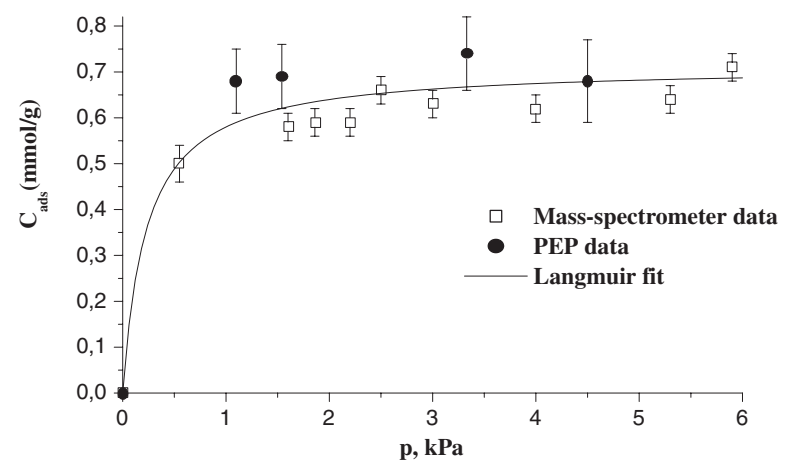

Figure 3. Isotherm of 3-methylpentane adsorption in silicalite at $403 \mathrm{~K}$. 


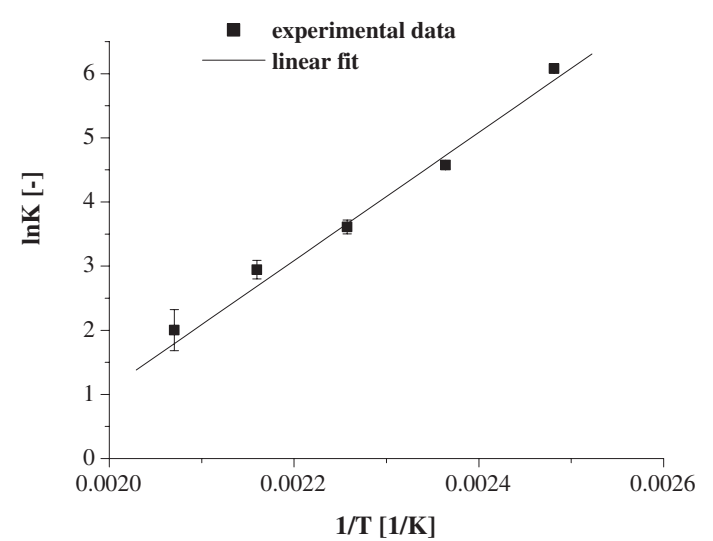

Figure 4. Arrhenius plot of fitted Langmuir adsorption constants for 3-methylpentane in silicalite.

tion has been determined to be $65 \pm 5 \mathrm{~kJ} / \mathrm{mol}$. The heat of adsorption obtained experimentally by different techniques are of the same value: 66.4 (inverse chromatography technique [26]), 60 (wall-coated capillary chromatographic method [27]), and $62.7 \mathrm{~kJ} / \mathrm{mol}$ (gravimetric sorption uptake method [25]). Theoretical calculations from June et al. [28] provided the value of $63 \mathrm{~kJ} /$ mol, which also supports the experimental data of this study. Thus, the adsorption isotherm data measured in this work agree with those from other techniques and will be further used for the diffusion data interpretation.

\subsubsection{Concentration dependence of the diffusivity}

The self-diffusivity of 3-methylpentane in silicalite is shown in figure 5 as a function of loading. The diffusivity monotonically decreases with the loading, which corresponds to the type-I concentration dependence [7] as mentioned in the Introduction. Quite an opposite effect of the pore occupancy was observed by the Inverse Chromatography technique [26] for 3methylpentane as well as for isopentane and 2,2dimethylbutane. Most probably, this was due to the small size $(2 \mu \mathrm{m})$ of silicalite crystals used in the experiments, so the process was not controlled by the intracrystalline diffusion.

As we have discussed in the Introduction, the decrease in the diffusivity with the pore occupancy was observed for other hydrocarbons in zeolites and predicted from some theoretical simulations. The type of concentration dependence (see Introduction) is mainly determined by the zeolite-adsorbate and adsorbate-adsorbate interactions [7]. Dynamic Monte Carlo simulations and mean-field studies performed by Coppens et al. [3] recovered all five types of diffusional behavior and it was shown that in silicalite, owing to the absence of interactions with acid sites, a monotonous decrease of the self-diffusion coefficient can be observed. This is in agreement with our observations as well as with the results of MD simulations performed by Schuring et al. [13] that showed a decrease in the diffusivity for $n$-butane in silicalite.

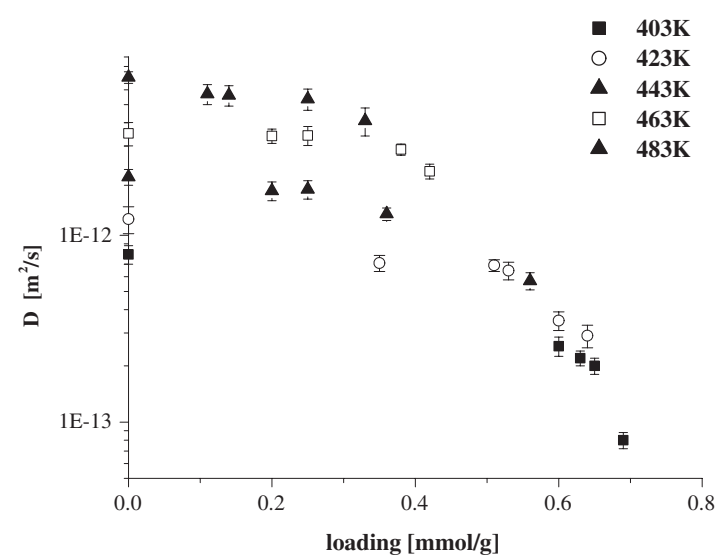

Figure 5. Concentration dependence of the diffusivity of 3-methylpentane in silicalite.

In general, the diffusion mechanism is considered to be a sequence of activated jumps from one adsorption site to another. Activated diffusion is described by the Arrhenius-type equation:

$$
D=D_{0} \cdot \exp \left(-\frac{E_{a}}{R T}\right)
$$

where $D_{0}$ is a preexponential factor, $E_{a}$ is the activation energy of diffusion, $R$ is the gas constant, and $T$ the temperature. The preexponential factor $D_{0}$ is related to a jump frequency between adsorption sites in the zeolite lattice [29], while the exponential expresses the chance that the molecules are able to overcome the free energy barrier $E_{a}$ between these sites.

Observed decrease of the self-diffusion coefficient with the pore occupancy might be caused by the concentration dependence of the activation energy of diffusion or by decrease of the jump frequency. According to the MD simulations [13], the potential energy of the alkane-alkane interaction increases monotonically with the loading, while the interaction between the alkane and zeolite lattice remains approximately constant. This means that the diffusivity in silicalite decreases because molecules hinder each other at high pore occupancies and the activation energy does not change with the occupancy. Mean-field theory predicted a very general behavior for the molecules in zeolites: a molecule can only hop to a neighbor site if this site is vacant, the average probability of this is $(1-\theta)$, where $\theta$ is the pore occupancy. Thus, a linear decrease in the diffusion with the loading can be expected: $D(\theta)=D(0) \cdot(1-\theta)$, where $D(\theta), D(0)$ are the self-diffusivities at $\theta$ and zero loadings, respectively. Lately, a number of theoretical studies have shown that a negative deviation from this linearity can be expected. One of the main arguments is that the molecular displacements are correlated to each other and zeolite topology has to be taken into account [12]. Indeed, Coppens et al. [12] showed that the self-diffusivity in MFI-type zeolites decreases even stronger with the 
occupancy because of the low lattice connectivity. The best approximation describing the concentration dependence of the diffusion coefficient calculated from dynamic Monte Carlo simulations would be a stretched exponential. Earlier, Theodorou et al. [15] modeled diffusion in ZSM-5 zeolite as a random walk process in a two-dimensional pore network and a deviation from the linear dependence similar to the one revealed by Coppens et al. [12] was also observed. Tsikoyiannis and Wei [16] used Monte Carlo simulations to calculate the dependence of the self-diffusivity on the occupancy and they found for the cubic and square lattices that the dependence is described by a function that lies somewhere between $(1-\theta)$ and $(1-\theta)^{2}$.

The experimentally obtained dependence (type-I [7]) of the diffusivity of 3-methylpentane in silicalite shown in figure 5 cannot unambiguously be described as linear, and the deviation discussed above is not likely to be observed. This could be due to the limited experimental accuracy. At lower temperatures, a bit slower than linear decrease in the diffusivity with the pore occupancy was observed, while at a higher temperature $T=483 \mathrm{~K}$, it could be described as linear. Thus, from figure 5 , it is difficult to draw clear conclusion about the function most suitably describing the experimental data.

Nevertheless, it is important to establish whether the activation energy of diffusion is increasing with the pore occupancy of the zeolite, thus causing the observed decrease in the diffusion.

\subsection{Activation energy of diffusion}

\subsubsection{Influence of the loading}

In this section, the factors influencing the activation energy of diffusion, such as partial pressure and zeolite pore occupancy, will be discussed. In some particular cases, activation energy was influenced by the zeolite loading. Indeed, MD simulations performed by Schuring et al. [13] for $n$-butane in mordenite demonstrated that with an increased pore loading the potential energies of alkane-lattice and alkane-alkane interactions change, and an increase in the activation energy of diffusion is observed. A double increase in the activation energy of diffusion occurred for $n$-butane in mordenite from the MD simulations, when the pore occupancy reached 0.8 from the maximum loading [13].

In order to investigate the influence of the silicalite pore occupancy, the activation energies of diffusion measured at two significantly different degrees of pore filling have been compared. Zero and approximately $50 \%$ pore occupancies were used. In order to measure the diffusivities at a fixed loading, partial pressures were chosen so as to ensure that at all temperatures it was fixed at approximately $0.35 \mathrm{mmol} / \mathrm{g}$. The value of the partial pressure of 3-methylpentane corresponding to

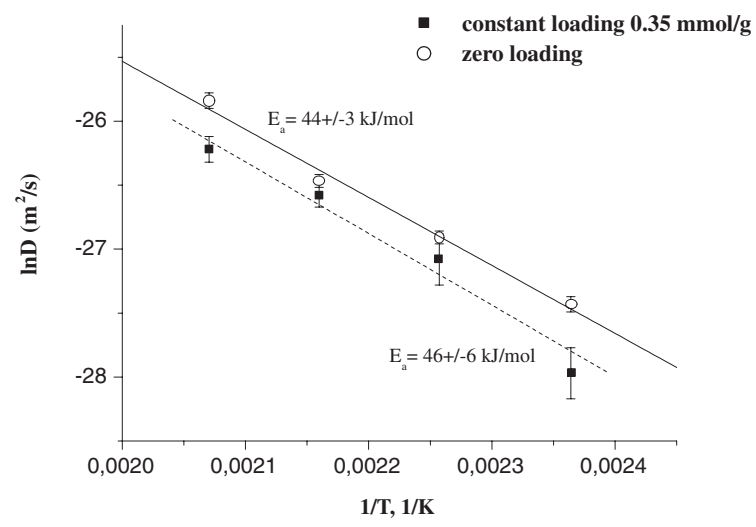

Figure 6. Arrhenius plots for the diffusivity of 3-methylpentane in silicalite at zero and constant occupancy of approximately 0.47 from the maximum loading.

the particular loading was obtained from the isotherms of adsorption (see Section 4.1).

In figure 6, the Arrhenius plots obtained for both pore occupancies are shown. The activation energies of diffusion determined from the slope of the Arrhenius plot at high and zero loadings are equal to $46 \pm 6 \mathrm{~kJ} / \mathrm{mol}$ and $44 \pm 4 \mathrm{~kJ} / \mathrm{mol}$ respectively, meaning that for 3-methylpentane in silicalite, the activation energy does not depend on the pore occupancy. Heink et al. [30], using PFG NMR, observed a similar picture for $C_{3}-C_{6} n$-alkanes in silicalite. For these alkanes, the selfdiffusivities decrease with the loading, while the activation energies were found to be independent.

The Arrhenius plots in figure 6, corresponding to different loadings ( 0 and $0.35 \mathrm{mmol} / \mathrm{g}$, respectively) are parallel to each other, so that the pre-exponential factor $D_{0}$ is smaller for high pore occupancy than that for the empty zeolite (smaller value of the intercept $\left(\ln D_{0}\right.$ ) corresponds to a higher loading). Similar experiments have been performed for $C_{1}-C_{6} n$-alkanes in silicalite with PFG NMR [30]. For those alkanes, the activation energy did not change, while the preexponential factor decreased as the zeolite loading changed from $1 / 4,1 / 2$, $3 / 4$, and 1 molecule per unit cell. Apparently, the concentration dependence of the self-diffusion coefficient is related to the change in the jump frequency but not to the activation energy. Therefore, the preexponential factor (equation 8) depends on the pore occupancy, which probably means that with increased occupation of the adsorption sites the jump frequency decreases because the probability of the successful jump decreases.

Comparison with literature data. The influence of the concentration on the diffusivity makes the comparison of the diffusional parameters with the literature more difficult because the experimental conditions might be the reason for the discrepancies. The most common expression used to extract self-diffusion coefficients from the transport diffusivities obtained from 
macroscopic measurement of uptake and permeation rates, or from the analysis of frequency responses, is the Darken equation:

$$
D_{t}(\theta)=D_{c} \frac{\partial \ln p}{\partial \ln \theta},
$$

where $D_{t}$ is the measured transport diffusivity, $D_{c}$ is the corrected diffusivity and $p$ is the partial pressure of a compound. Values of $D_{c}$ are used to compare with the self-diffusivities, which is not really correct as was shown by Maginn et al. [31] and recently by Paschek and Krishna [32]. In these studies, the discrepancies between the self-diffusion coefficient and the corrected diffusivity at nonzero pore occupancies have been demonstrated. Linear dependence of the corrected diffusivity on the pore occupancy $(1-\theta)$ and stronger decrease in selfdiffusion for methane and 2-methylhexane in silicalite were observed using kinetic Monte Carlo simulations performed by Paschek and Krishna [32]. Therefore, when comparing the diffusivities obtained by different methods, one should keep in mind that both corrected and self-diffusivities are concentration dependent and are not necessarily close to each other.

The self-diffusivity of 3-methylpentane in silicalite measured here have been compared with those from other methods determined under close conditions (zeolite loading and temperature) and are shown in table 1. Apparently, diffusivities measured with the PEP technique are in reasonable agreement with those from other studies, except for the self-diffusion coefficient determined by Inverse Chromatography technique [26], which is significantly lower compared to others. The reason for this disagreement is not clear.

The values of the activation energy of 3-methylpentane diffusion in silicalite measured by different authors are shown in table 2 . The activation energy obtained from the corrected diffusivities by Millot et al. using the Membrane permeation technique [33] is equal to $50 \mathrm{~kJ} /$ mol, which is quite close to the value of the activation energy measured here. Deviation from the linear dependence was observed for the logarithm of the diffusivity versus reciprocal temperature, which was attributed by the authors to the strong variation of diffusion coefficient with the coverage.

In the literature, one can find activation energy values that are essentially different from each other. Gravi- metric measurements on large crystals $(220 \mu \mathrm{m})$ gave the corrected activation energy equal to $58 \mathrm{~kJ} / \mathrm{mol}$ [34], partial pressure conditions were not specified. Twotimes lower activation energy $(29 \mathrm{~kJ} / \mathrm{mol})$ was found by the wall-coated capillary chromatographic method on small crystals $(2 \mu \mathrm{m})$ [27]. Inverse Chromatography experiments performed on small crystals $(2 \mu \mathrm{m})$ provided the activation energy of diffusion at zero occupancy of $56 \mathrm{~kJ} / \mathrm{mol}$ [26], which is slightly higher than measured in this study. Modeling of the data used in the Inverse Chromatography method implies intracrystalline diffusion limitations, which probably was not the case, since the size of silicalite crystals used in the study [26] was not big enough. Probably, owing to the absence of the intracrystalline diffusion limitations, the increase in the diffusivity with the loading was observed as well as the deviation of the data from the Arrhenius dependence. Zhu et al. [35] calculated $52.4 \mathrm{~kJ} / \mathrm{mol}$ as the value for the activation energy of 3-methylpentane diffusion from the corrected diffusivities measured by the tapered element oscillating microbalance (TEOM) technique on large silicalite crystals $(120 \mu \mathrm{m})$. In this study [35], the activation energy was obtained from the corrected diffusivities measured at different partial pressure and pore occupancy conditions. From TEOM data [35], we have chosen the diffusivities measured at similar loadings (approximately $3.8 \mathrm{~mol} /$ u.c.) at different temperatures to estimate the so-called true activation energy that is determined under constant loading to exclude its influence. The value obtained turned out to agree very well with the activation energy measured in this study: $46 \pm 3 \mathrm{~kJ} / \mathrm{mol}$ and $44 \pm 3 \mathrm{~kJ} / \mathrm{mol}$ (zero loading), from TEOM and PEP, respectively.

Therefore, owing to the concentration dependence of the self-diffusivity, the true activation energy of diffusion can only be measured at zero pore occupancy or under constant loading conditions in order to eliminate a possible influence of the experimental conditions.

\subsubsection{Influence of the partial pressure}

In the macroscopic experiments, the activation energy is usually measured under constant partial pressure of the component. This has also been done in this work within the temperature range $T=403-483 \mathrm{~K}$.

As the pressure increases from 0 to $4.5 \mathrm{kPa}$ (figure 7), an unusual effect of the partial pressure of 3-methyl-

Table 1

Activation energies of 3-methylpentane diffusion in silicalite obtained by different methods

\begin{tabular}{lccl}
\hline Method & $D \times 10^{-13}, \mathrm{~m}^{2} / \mathrm{s}$ & Conditions & \multicolumn{1}{c}{ Type of $D$} \\
\hline PEP & 3.1 & $423 \mathrm{~K}, 4.5 \mathrm{kPa}$ & Self-diffusion \\
PEP & 2.9 & $403 \mathrm{~K}, 1.3 \mathrm{kPa}$ & Self-diffusion \\
Membrane permeation [33] & 2.47 & $423 \mathrm{~K}, 0.1 \mathrm{bar}$ & Intraparticular \\
Gravimetry [34] & 1.1 & $373 \mathrm{~K},-$ & Corrected \\
Inverse chromatography [26] & 0.007 & $423 \mathrm{~K}, 0$ & Effective \\
TEOM [35] & $4.20-4.71$ & $408 \mathrm{~K}, 0.94-6.35 \mathrm{kPa}$ & Corrected \\
\hline
\end{tabular}


Table 2

Activation energies of 3-methylpentane diffusion in silicalite obtained by different methods

\begin{tabular}{lcc}
\hline Method & $E_{a}, \mathrm{~kJ} / \mathrm{mol}$ & Crystal size, $\mu \mathrm{m}$ \\
\hline PEP & $44 \pm 3$ & 150 \\
Membrane permeation [33] & 50 & 0.5 \\
Gravimetry [34] & 58 & 220 \\
Wall-coated capillary chromatography [27] & 29 & 2 \\
Inverse chromatography [26] & 56 & 2 \\
TEOM [35] & 52.4 & 120 \\
\hline
\end{tabular}

pentane on the apparent activation energy of diffusion was observed: a significant rise in the activation energy from 44 to $80 \mathrm{~kJ} / \mathrm{mol}$ occurred. The values of the apparent activation energy measured at different partial pressures of alkane are shown in table 3 .

Significantly lower activation energies $(50 \mathrm{~kJ} / \mathrm{mol})$, compared to the values reported here for similar conditions, were measured at 0.1 bar and at 0.06 bar of feed pressure with the Membrane permeation technique [33]. Corrected diffusivities from [33] were almost equal at these partial pressures, which means that there was no concentration effect observed. Probably, this is due to the partial pressure conditions that were high enough so that the diffusivity did not change much anymore.

We believe that the observed effect of the partial pressure on the apparent activation energy (figure 7) is a consequence of the concentration dependence of the

Table 3

Apparent activation energies of 3methylpentane diffusion in silicalite at different partial pressures

\begin{tabular}{lc}
\hline Pressure, $\mathrm{kPa}$ & $E_{a}, \mathrm{~kJ} / \mathrm{mol}$ \\
\hline 0 & $44 \pm 3$ \\
1.33 & $65 \pm 4$ \\
2.00 & $61 \pm 3$ \\
3.70 & $70 \pm 5$ \\
4.50 & $80 \pm 6$ \\
\hline
\end{tabular}

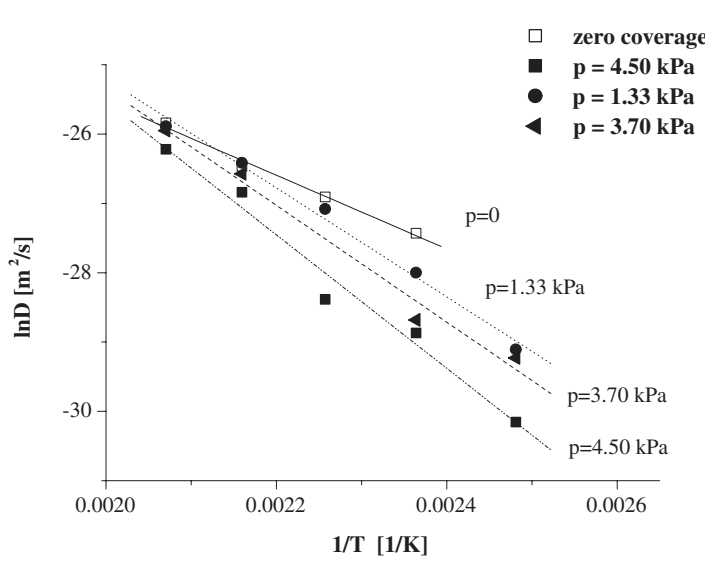

Figure 7. Influence of the partial pressure on the 3-methylpentane activation energy of diffusion in silicalite. self-diffusivity. As the concentration inside zeolite pores under given partial pressure depends on the temperature, the combined effect of temperature and loading dependence is measured. With increasing temperature the loading decreases. According to the observed effect of the loading on the diffusion coefficient (figure 5), in addition to the increased mobility of the molecules due to the higher temperature, diffusivity also increases because of the decreased loading, which is caused by the increase in the jump frequency $D_{0}$. As a result, the apparent activation energy can be much higher then the real activation energy, and this value depends on the hydrocarbon partial pressure, at which the measurements are performed.

\subsection{Concentration dependence of the diffusivity}

It was experimentally established that for 3-methylpentane in silicalite, the dependence of the apparent activation energy of diffusion on the partial pressure is caused by the concentration dependence of the preexponential factor $D_{0}$. Therefore, from the diffusivities at given loadings and the true activation energy, we can estimate the concentration dependence of $D_{0}$ :

$$
D_{0}=D(\theta) \cdot \exp \left(\frac{E_{a}}{R T}\right)
$$

where $D_{0}(\theta)$ is the pre-exponential factor that corresponds to the self-diffusivity $D(\theta)$ at pore occupancy $\theta$ and temperature $T$, and $E_{a}$ is activation energy measured at zero loading.

Several predictions have been made on the concentration dependence:

(1) Mean-field theory predicted a linear decrease in the self-diffusivity proportional to $(1-\theta)$, which is the probability factor for the molecule to jump into the neighboring adsorption site [14]. It does not take into account zeolite topology or specific interactions between the molecule and zeolite:

$$
D(\theta)=D(0) \cdot(1-\theta),
$$


with the diffusivity at zero pore occupancy $D(0)$ :

$$
D(0)=D_{\infty} \cdot \exp \left(-\frac{E_{a}}{R T}\right)
$$

Thus, the overall equation for the self-diffusion coefficient is

$$
D(\theta)=D_{\infty}(1-\theta(T)) \cdot \exp \left(-\frac{E_{a}}{R T}\right)
$$

In this expression, $D_{\infty}$ is a self-diffusion coefficient at infinite temperature in the zeolite with zero loading. Therefore, the pre-exponential factor $D_{0}(\theta)$ is expressed from equations 10,11 , and 13 as follows:

$$
D_{0}(\theta)=D_{\infty} \cdot(1-\theta) .
$$

(2) Kinetic Monte Carlo simulations predict deviations from the linear dependence for MFI-type zeolite due to its poorly connected lattice [12]. The average connectivity of silicalite is 2.67 if the molecule can jump to any adsorption site: zigzag, straight channels, or the intersections. In this particular case of 3-methylpentane in silicalite, the connectivity is 4, because according to Vlugt et al. [24] channel, intersections are the most favorable locations for monobranched hexanes. Only very high pressure is required for the molecules to adsorb in the channels [24]. For this connectivity, the deviation from the linear dependence is expected to be very small [12]. Simulated dependence can be approximated in general by a stretched exponential:

$$
\begin{aligned}
D(\theta) & =D(0) \cdot \exp (-b \cdot \theta), \\
D_{0}(\theta) & =D_{\infty} \cdot \exp (-b \cdot \theta),
\end{aligned}
$$

where $b$ is a fitted coefficient that depends on the connectivity for the molecule/zeolite system. The self-diffusivity at zero coverage depends on the dimension of the lattice only, because there are no interactions between the molecules.

(3) From calculations based on transition state theory, a general formula was derived to describe the concentration dependence of surface and zeolitic diffusions [18]:

$$
\frac{D(\theta)}{D(0)}=\frac{1-\theta+\frac{1}{2} \lambda \theta(2-\theta)+H(1-\lambda) \cdot(1-\lambda) \frac{1}{2} \lambda \theta^{2}}{\left(1-\theta+\frac{1}{2} \lambda \theta\right)^{2}},
$$

$$
D_{0}(\theta)=D_{\infty} \frac{1-\theta+\frac{1}{2} \lambda \theta(2-\theta)+H(1-\lambda) \cdot(1-\lambda) \frac{1}{2} \lambda \theta^{2}}{\left(1-\theta+\frac{1}{2} \lambda \theta\right)^{2}} .
$$

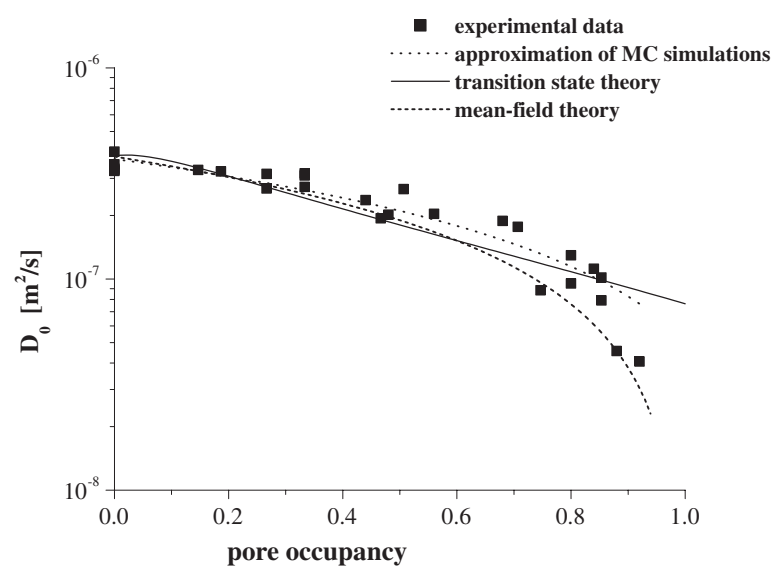

Figure 8. A plot of pre-exponential factor $D_{0}$ for 3-methylpentane diffusion in silicalite versus zeolite loading. The data were fitted by theoretically predicted dependencies.

In this expression, $\lambda$ is a blockage parameter and is equal to the ratio between the rate constant of return migration due to blockage by another molecule and the rate constant of forward migration. $\lambda$ is equal to zero for surface diffusion and $\lambda>0$ for zeolitic diffusion. Proximity of the sizes of adsorbate and zeolite pore should result in large values of $\lambda$. $H(1-\lambda)$ is the Heaviside step function. Expression 17 was claimed to describe all five possible types of concentration dependence of diffusion in zeolites described in work of Kärger and Pfeifer, [7].

Figure 8 shows the attempt to fit the pre-exponential factor calculated from the experimental data according to equation 10, with the functions 14, 16, and 18 described above. In table 4 , one can find the characteristics of fitting: $\chi^{2}$ chi square value of fit and Coefficient of Determination $\left(R^{2}\right)$. The minimum value of $\chi^{2}$ and higher value of $R^{2}$ correspond to a better fit.

Apparently, the stretched exponential approximation predicted by Kinetic Monte Carlo simulations [12] seems to be a slightly better fit for the experimental data: first-order decay with the fitted coefficient $b=1 / 3.239$. Even though expression 17 failed to describe the data for 3-methylpentane in silicalite, the fitted value of $\lambda=10.02$ is in line with the values found by Chen and Yang [18] for the diffusion of thriethylamine in 13X zeolite and for benzene in ZSM-5, that are equal to 10.2 and 2.11 respectively.

According to Coppens [12], the difference between mean-field theory and Monte Carlo simulations should Table 4

Fitting characteristics for the functions predicted by theoretical calculations

\begin{tabular}{lll}
\hline Function & \multicolumn{1}{c}{$\chi^{2}$} & $R^{2}$ \\
\hline Mean-field & $1.6558 \cdot 10^{-15}$ & 0.85388 \\
Monte Carlo & $1.07623 \cdot 10^{-15}$ & 0.91233 \\
Transition state theory & $1.7439 \cdot 10^{-15}$ & 0.8078 \\
\hline
\end{tabular}


be insignificant for the investigated system, which is indeed observed here. A stronger decrease in the selfdiffusion with the occupancy is caused by the higher probability of the return jump, because the probability for a previously visited site to be vacant is higher than that for any other site. On the contrary, mean-field theory predicts that the probability to hop to any of the neighbor sites is equal and zeolite topology is not considered. This is a main reason for the contradictions in these two theories. According to kinetic Monte Carlo simulations, the probability of the successful jump also depends on the connectivity between the adsorption sites. For the intersections, the connectivity factor is higher, which makes the deviations from the mean-field theory dependence smaller. Indeed, one can see from the fitting (figure 8) that both functions are quite close to each other. Unfortunately, there is no possibility of comparing the obtained coefficient $b$ with the literature, since the exponential dependence is just an approximation of the simulated effect of the zeolite loading on the diffusion coefficients. Nevertheless, we tried to compare the approximated dependencies with those for the diamond lattice that also has a dimension of 3 and connectivity of 4 , which is similar to 3-methylpentane in silicalite. In the diamond lattice, the deviations from a linear dependence are very insignificant [12] and the fitting provides a coefficient $b=1 / 2.753$. The more essential are the correlation effects, so that the diffusivity decreases faster with the concentration, the bigger the value of $b$. Apparently, for the studied case, the deviations from the linear dependence of the diffusion coefficient on the pore occupancy are within the experimental accuracy. Only based on the data analysis, one may conclude that a slight deviation from the linear dependence is observed.

\section{Conclusions}

Using the PEP technique, the concentration dependence of self-diffusivity of 3-methylpentane in silicalite has been studied. The self-diffusion coefficient can best be described as monotonously decreasing as the pore occupancy increases, which corresponds to type-I of the concentration dependence according to Kärger and Pfeifer [7]. Since the activation energy of diffusion of the alkane turned out to be independent on the pore occupancy, the pre-exponential factor $D_{0}$ is concentration dependent. For molecular diffusion in silicalite, theoretical studies predict a linear decrease of the diffusion coefficient $D(\theta)=D(0) \cdot(1-\theta)$ (mean-field theory) and negative deviation from linear behavior, which can be approximated by a stretched exponential $D(\theta)=D(0) \cdot \exp (-b \cdot \theta)$, where $b$ is a coefficient for a particular system (approximation of Monte Carlo simulations). Analysis of the experimental data showed that the stretched exponential is a slightly better fit for the experimental data. Moreover, the theoretically predicted deviations from the linear dependence are also very small for this system. Since 3-methylpentane molecules most probably jump to the channel intersections only, the connectivity of the system is 4, which leads to a smaller deviation from the linear dependence.

As a consequence of the concentration dependence of the pre-exponential factor $D_{0}$, the apparent activation energy increases with partial pressure. Usually, in the macroscopic experiments, the measurement of the activation energy is performed under fixed partial pressure conditions. If the conditions are different from those used in other studies, it can be the reason for the discrepancies in the apparent activation energy and diffusivities obtained by different authors. Values of activation energy of diffusion should be compared only when measured at zero pore filling or under fixed loading conditions.

\section{Acknowledgments}

The authors are grateful to Shell Research and Technology Center (Amsterdam) for the silicalite sample. Financial support from NWO-Spinoza grant made this work possible.

\section{References}

[1] J. Kärger and D.M. Ruthven, Diffusion in Zeolites and Other Microporous Solids (John Wiley \& Sons, New York, 1992).

[2] D. Schuring, A.O. Koriabkina, A.M. de Jong, B. Smit and R.A. van Santen, J. Phys. Chem. B 105(32) (2001) 7690

[3] M.-O. Coppens, A.T. Bell and A.K. Chakraborty, Chem. Eng. Sci. 54 (1999) 3455.

[4] B.G. Anderson, F.M.M. de Gauw, N.J. Noordhoek, L.J. van IJzendoorn, R.A. van Santen and M.J.A. de Voigt, Ind. Eng. Chem. Res. 37(3) (1998) 815.

[5] A. Dyer and G.E. Moores, J. Inorg. Nucl. Chem. 43(9) (1981) 2146.

[6] K.A. Vonkeman, G. Jonkers and R.A. van Santen, Stud. Surf. Sci. Catal. 71 (1991) 239.

[7] J. Kärger and H. Pfeifer, Zeolites 7 (1987) 90.

[8] M. Bülow, H. Schodder, L.V.C. Rees, J. Caro and R. Richards, Proc. of 7th International Zeolite Conference (Tokyo, 1986).

[9] N. van den Begin, L.V.C. Rees, J. Caro and M. Bülow, Zeolites 9 (1989) 287.

[10] A. Micke, M. Bülow, M. Kočirík and P. Struve, J. Phys. Chem. B 98 (1994) 12337.

[11] J. Caro, M. Bülow, W. Schimer, J. Kärger, W. Heink and H. Pfeifer, J. Chem. Soc., Faraday Trans. 81 (1985) 2541.

[12] M.-O. Coppens, A.T. Bell and A.K. Chakraborty, Chem. Eng. Sci. 53 (1998) 2053.

[13] D. Schuring, A.P.J. Jansen and R.A. van Santen, J. Phys. Chem. B 2000 (104) 941.

[14] J. Jost, Diffusion in Solids, Liquids, Gases (Academic Press, New York, 1960).

[15] W. Theodorou and J. Wei, J. Mol. Catal., A: Chem. 83 (1983) 205.

[16] J.G. Tsikoyiannis and J. Wei, J. Chem. Eng. Sci. 46 (1991) 233.

[17] B.L. Trout, A.K. Chakraborty and A.T. Bell, Chem. Eng. Sci. 52(14) (1997) 2265.

[18] Y.D. Chen and R.T. Yang, AIChE J. 37(10) (1991) 1579. 
[19] R.H. Cunningham, A.V.G. Mangnus, J. van Grondelle and R.A. van Santen, J. Mol. Catal., A: Chem. 107 (1996) 153.

[20] A.V.G. Mangnus, L.J. van IJzendoorn, J.J.M. de Goeij, R.H. Cunningham, R.A. van Santen and M.J.A. de Voigt, Nucl. Instrum. Methods, Sect. B 99 (1995) 649.

[21] N.J. Noordhoek, L.J. van IJzendoorn, B.G. Anderson, F.M.M. de Gauw, R.A. van Santen and M.J.A. de Voigt, Ind. Eng. Chem. Res. 37(3) (1998) 825.

[22] W.E. Schiesser, The Numerical Method of Lines (Academic Press, San Diego, CA, 1991).

[23] R.A. van Santen, B.G. Anderson, R.H. Cunningham, A.V.G. Mangnus, J. van Grondelle and L.J. van IJzendoorn, Stud. Surf. Sci. 101 (1996) 791.

[24] T.J. Vlugt, R. Krishna and B. Smit, J. Phys. Chem. B 103 (1999) 1102.

[25] C.L. Cavalcante and D.M. Ruthven, Ind. Eng. Chem. Res. 34 (1995) 177.

[26] E. Jolimaitre, M. Tayakout, C. Jallut and K. Ragil, Ind. Eng. Chem. Res. 40 (2001) 914.
[27] M.A. Jama, M.P.F. Delmas and D.M. Ruthven, Zeolites 18 (1997) 200.

[28] R.L. June, A.T. Bell and D.N. Theodorou, J. Phys. Chem. B 94 (1990) 1508.

[29] N.Y. Chen, T. Degnan and C. Smith, Molecular Transport and Reaction in Zeolites (John Wiley \& Sons, New York, 1994).

[30] W. Heink, J. Kärger, H. Pfeifer, K.P. Datema and A.K. Nowak, J. Chem. Soc., Faraday Trans. 88 (1992) 3505.

[31] E.J. Maginn, A.T. Bell and D.N. Theodorou, J. Phys. Chem. B 97 (1993) 4173.

[32] D. Paschek and R. Krishna, Chem. Phys. Lett. 333 (2001) 2784.

[33] B. Millot, A. Méthivier, H. Jobic, H. Moueddeb and J.A. Dalmon, Microporous Mesoporous Mater. 38 (2000) 85.

[34] C.L. Cavalcante and D.M. Ruthven, Ind. Eng. Chem. Res. 34 (1995) 185.

[35] W. Zhu, F. Kapteijn and J.A. Moulijn, Microporous Mesoporous Mater. 47 (2001) 157. 